\title{
Vigilancia molecular de aislamientos invasores de Streptococcus pneumoniae resistentes a la penicilina en niños colombianos menores de 5 años
}

\begin{abstract}
Jaime Moreno, Vienvilay Phandanouvong, Elizabeth Castañeda
Grupo de Microbiología, Instituto Nacional de Salud, Bogotá, D.C., Colombia.

El incremento de aislamientos de Streptococcus pneumoniae resistentes a la penicilina se favorece por la presión selectiva del antibiótico y la dispersión clonal. Como parte del programa de vigilancia molecular, por medio del uso de la electroforesis en campo pulsado (PFGE), se determinaron las relaciones genéticas de 190 aislamientos invasores de $S$. pneumoniae con susceptibilidad disminuida a la penicilina recuperados de niños colombianos menores de 5 años durante los años 2000-2003. Se identificaron 42 diferentes patrones electroforéticos; cuatro patrones agruparon el $76 \%$ de los aislamientos, los cuales se encontraron relacionados con los clones internacionales 1-España ${ }^{23 F}$, 2-España ${ }^{6 B}$, 3-España ${ }^{9 \vee}$ y 26 -Colombia ${ }^{23 F}$. Nuestros resultados indican que la diseminación de $S$. pneumoniae resistente a la penicilina es el resultado de la dispersión de clones internacionales, especialmente, del clon 3-España ${ }^{9 v}$.
\end{abstract}

Palabras clave: Streptococcus pneumoniae, susceptibilidad disminuida a la penicilina, PFGE, clones, relaciones genéticas.

Molecular surveillance of invasive penicillin-resistant Streptococcus pneumoniae
Colombian isolates recovered from children less than 5 years of age

The rapid increase of penicillin-resistant Streptococcus pneumoniae isolates could be a consequence of the spread of clones or due to the antimicrobial selective pressure. The genetic relatedness of 190 invasive isolates $S$. pneumoniae with reduced susceptibility to penicillin recovered from Colombian children less than 5 years old during a surveillance study from 2000 to 2003 was determined by the use of pulsed-field electrophoresis (PFGE). Overall, 42 different PFGE patterns were identified, but 4 of them included $76 \%$ of all isolates. They were related with international clones 1-Spain ${ }^{23 F}, 2-S^{2}$ ain $^{6 \mathrm{~B}}, 3-$ Spain $^{9 \mathrm{~V}}$ and 26-Colombia ${ }^{23 \mathrm{~F}}$. Our results indicated that the dissemination of penicillin-resistant $S$. pneumoniae was the result of the spread of international clones, specially, the 3-Spain ${ }^{9 v}$ clone.

Key words: Streptococcus pneumoniae, diminished susceptibility to penicillin, PFGE, clone, genetic relatedness

Streptococcus pneumoniae es una de las principales causas de morbilidad y mortalidad en el mundo que, asociado con el desarrollo de resistencia a los antibióticos, se constituye en uno de los principales problemas de salud pública (1).

Correspondencia:

Elizabeth Castañeda, Grupo de Microbiología, Instituto Nacional de Salud, Avenida calle 26 No. 51-60, Bogotá, D.C., Colombia.

Teléfono: (571) 220 7700, extensión 445

ecastaneda@ins.gov.co

Recibido: 16/07/04; aceptado: 08/09/04
La implementación de programas de vigilancia en combinación con la aplicación de técnicas de tipificación molecular han permitido la identificación y el seguimiento de diversas líneas clonales de $S$. pneumoniae resistentes a la penicilina y a otros antibióticos, las cuales tienen amplia dispersión geográfica tanto regional como internacional (2-4).

En Colombia, en 1994, a través del proyecto del Sistema Regional de Vacunas (SIREVA) de la Organización Panamericana de la Salud, se inició un proyecto regional para la vigilancia de la 
distribución de los tipos capsulares y de la susceptibilidad antimicrobiana de los aislamientos de $S$. pneumoniae, causantes de enfermedad invasora en niños menores de 5 años (5). Los estudios moleculares de los aislamientos invasores con susceptibilidad disminuida a la penicilina (SDP) demostraron la circulación en Colombia de los clones internacionales 1$E^{2}$ spaña ${ }^{23 F}$, 2-España ${ }^{6 B}$, 3-España ${ }^{9 V}$ y 26Colombia $^{23 F}(6,7)$. El objetivo de este trabajo fue continuar la vigilancia molecular de los aislamientos invasores de $S$. pneumoniae con SDP recuperados de niños colombianos menores de 5 años procedentes de diferentes regiones del país durante los años de 2000 a 2003 para determinar los tipos clonales prevalentes y circulantes en esta población y relacionarlos con los serotipos, patrones de resistencia a los antibióticos y diagnóstico clínico.

\section{Materiales y métodos}

Aislamientos y características de los pacientes. Se estudiaron 190 aislamientos invasores de $S$. pneumoniae con SDP recuperados de niños menores de 5 años, procedentes de diferentes regiones del país y remitidos al Grupo de Microbiología como parte de los programas de meningitis bacteriana (MBA) e infección respiratoria aguda (IRA) de enero de 2000 a diciembre de 2003.

Los aislamientos se obtuvieron de hemocultivos (53\%), líquido cefalorraquídeo (35\%), líquido pleural $(6 \%)$ y de otros líquidos corporales $(6 \%)$. El $44 \%$ de los pacientes tenía diagnóstico de neumonía, $39 \%$ de meningitis y $17 \%$ de otros procesos invasores (sepsis, peritonitis). La mayoría de los aislamientos procedían de niños (57\%) y menores de 2 años (67\%).

Los aislamientos estaban serotipificados con la reacción capsular de Quellung. El serotipo 14 fue el predominante $(65 \%)$, seguido por los serotipos $23 \mathrm{~F}(15 \%)$ y $6 \mathrm{~B}(9 \%) ; 6 \%$ de los aislamientos presentaron el serotipo $9 \mathrm{~V}$ y $3 \%$ el $6 \mathrm{~A}$; los serotipos $19 \mathrm{~F}$ y 34 se presentaron en $1 \%$. A los aislamientos estudiados también se les había determinado la susceptibilidad antimicrobiana, expresada como la concentración inhibitoria mínima (CIM) en $\mu \mathrm{g} /$ $\mathrm{ml}$ a penicilina, ceftriaxona, eritromicina, cloranfenicol, trimetroprim-sulfametoxazol, vancomicina y tetraciclina. La metodología e interpretación se había realizado de acuerdo con las recomendaciones del Comité Nacional de Estándares para el Laboratorio Clínico (National Committee for Clinical Laboratory Standards, NCCLS) (8). En 50 aislamientos (26\%) se determinó resistencia intermedia a la penicilina $(\mathrm{CIM}=0,125-1,0 \mu \mathrm{g} / \mathrm{ml})$ y en $140(74 \%)$ resistencia alta $(\mathrm{CIM}>2,0 \mu \mathrm{g} / \mathrm{ml})$.

PFGE. Se realizó según el protocolo de Soares et al. (9) y las modificaciones de Vela et al. (7). Brevemente, los aislamientos se cultivaron en caldo Todd-Hewitt suplementado, hasta alcanzar la fase logarítmica de crecimiento. Las células fueron embebidas en discos de agarosa de bajo punto de fusión (Bio-Rad), digeridas con la enzima de restricción Smal (Promega) y los fragmentos de restricción se separaron en geles de agarosa al $1 \%$ (Bio-Rad) por electroforesis en campo pulsado usando el equipo CHEFF II (Bio-Rad). Los geles se colorearon con bromuro de etidio y se visualizaron con luz ultravioleta. Los patrones de las bandas se asignaron visualmente y se clasificaron según los criterios de Tenover (10); igualmente, se analizaron con el programa de cómputo Diversidad (Bio-Rad) para determinar el porcentaje de similitud genética de los aislamientos y obtener los dendrogramas correspondientes. Como cepas de control y referencia se emplearon la cepa R6, acapsular y sensible a antibióticos (donada por Alexander Tomasz de Rockefeller University, New York), los clones 1-España ${ }^{23 F}, 2-E^{2}$ spaña ${ }^{6 \mathrm{~B}}$ y 3-España ${ }^{9 \mathrm{~V}}$ (suministrados por el Centro Nacional de Streptococcus, NCS, Alberta, Canadá) y los aislamientos colombianos INS Spn E-147 (representante del clon 26-Colombia ${ }^{23 F}$ ) y Spn 1206, serotipo 14 con patrón de PFGE relacionado con el clon 3-España ${ }^{9 v}(7)$.

Análisis estadístico. Con el fin de determinar una posible relación entre los grupos clonales y el diagnóstico clínico de los pacientes, se utilizó el programa Epilnfo 6.04 para la comparación de porcentajes determinando el valor de $p$. Se consideró una diferencia estadísticamente significativa un valor de $p<0,05$. 


\section{Resultados}

Con la técnica de electroforesis en campo pulsado y teniendo en cuenta la distribución por serotipos, los 190 aislamientos se agruparon en 42 patrones electroforéticos. Los patrones asignados como $A$, B, C y D agruparon el $76 \%$ de los aislamientos, 6 patrones (J-Ñ) $(7 \%)$ tenían 20 más aislamientos y los 32 restantes (17\%) un solo aislamiento.

\section{Serotipo $23 F$}

De los 29 aislamientos con tipo capsular 23F, 9 fueron multirresistentes y conformaron el patrón A relacionado por fenotipo y genotipo (relación genética $>89 \%$ ) con el clon 1-España ${ }^{23 F}$. El patrón A se subdividió en 8 subtipos, de los cuales $A$, A1 y A3 se habían informado previamente $(6,7)$. Además, 17 aislamientos con resistencia intermedia a la penicilina y variable a trimetroprimsulfametoxazol y tetraciclina (cuadro 1) y un aislamiento con serotipo 19F integraron el patrón C con 11 subtipos, que incluían C1 a C5 observados anteriormente en la población infantil colombiana $(6,7)$; los aislamientos con patrón C tenían una relación genética mayor del $82 \%$ con el clon 26-Colombia ${ }^{23 F}$. Los 3 aislamientos restantes presentaron patrones electroforéticos denominados $E, F$ y $G$ y no se asociaron con clones internacionales.

\section{Serotipos 14 y $9 \mathrm{~V}$}

Los aislamientos con serotipos $14(n=123)$ y $9 \mathrm{~V}$ $(n=11)$ se analizaron conjuntamente debido a que entre estos serotipos es un evento relativamente frecuente el intercambio de genes capsulares (11). 97 aislamientos con serotipo 14 y 9 con tipo capsular 9V conformaron el patrón B con 39 subtipos y se encontraron relacionados fenotípica y genotípicamente con el clon 3-España ${ }^{9 \mathrm{~V}}$ (similitud genética $>76 \%$ ). Los aislamientos se distribuyeron en los subtipos $B(n=4), B 1(n=12)$, B3 $(n=31), B 4(n=12), B 13(n=6), B 32(n=3)$, los subtipos B6, B12, B18, B26 y B37 con 2 aislamientos cada uno, y los restantes 28 subtipos tenían sólo un aislamiento. El subtipo B13 se observó solamente en los aislamientos recuperados en Bogotá. A diferencia de 3 aislamientos serotipo 14 con resistencia intermedia a la penicilina que integraron el patrón $\mathrm{H}$, los restantes aislamientos serotipo $14(\mathrm{n}=23)$ y $9 \mathrm{~V}(\mathrm{n}=2)$ tenían patrones electroforéticos no relacionados entre sí, ni con clones internacionales.

\section{Serotipo 6B}

De los 17 aislamientos con serotipo 6B, 14 integraron el patrón $D$ con 8 subtipos, fenotipo multirresistente (cuadro 1) y relacionados con el clon 2-España ${ }^{6 B}$ con un porcentaje de similitud genética mayor del $81 \%$. Los otros 3 aislamientos presentaron diversos patrones de PFGE.

\section{Otros serotipos}

Los 5 aislamientos con tipo capsular $6 \mathrm{~A}$ se distribuyeron en 3 patrones electroforéticos; J $(n=2)$, AK $(n=1)$ y $L(n=2)$; los serotipo $19 F$, uno se agrupó en el patrón $C$ y los otros 2 conformaron el patrón AL; por último, los aislamientos con tipo capsular $34(n=2)$ integraron el patrón AM.

\section{Diagnóstico y clonalidad}

Los aislamientos relacionados con los clones internacionales $1,2,3$ y 26 se recuperaron en el

Cuadro 1. Resistencia a los antibióticos según la relación clonal de los aislamientos invasores de $S$. pneumoniae con susceptibilidad disminuida a la penicilina recuperados de niños menores de 5 años.

\begin{tabular}{|c|c|c|c|c|c|c|c|c|c|c|}
\hline \multirow[t]{3}{*}{ Clon } & \multirow[t]{3}{*}{$\mathbf{n}$} & \multicolumn{7}{|c|}{$\%$ resistencia a los antibióticos } & \multirow{3}{*}{$\mathbf{E}$} & \multirow{3}{*}{ Tet } \\
\hline & & \multicolumn{2}{|c|}{$\mathbf{P}$} & \multicolumn{2}{|c|}{ Cro } & \multirow[t]{2}{*}{$\mathrm{Cl}$} & \multicolumn{2}{|c|}{ STX } & & \\
\hline & & Alta & $\mathbf{I}$ & Alta & $\mathbf{I}$ & & Alta & I & & \\
\hline 1-España ${ }^{23 F}$ & 9 & 100 & - & 67 & 33 & 100 & 100 & - & 11 & 100 \\
\hline 2-España ${ }^{6 B}$ & 14 & 93 & 7 & 28 & 72 & 64 & 100 & - & 64 & 86 \\
\hline 3-España ${ }^{9 v}$ & 106 & 93 & 7 & 19 & 81 & - & 98 & 2 & 1 & 7 \\
\hline 26-Colombia $23 F$ & 18 & - & 100 & - & - & - & 67 & 33 & - & 28 \\
\hline
\end{tabular}

P: penicilina, Cro: ceftriaxona, Cl: cloranfenicol; STX: trimetroprim-sulfametoxazol; E: eritromicina; Tet: tetraciclina Alta: resistencia alta, I: resistencia intermedia. 
$82 \%$ de los pacientes con neumonía y en el $70 \%$ de los pacientes con meningitis. En el cuadro 2 se observa la distribución de los aislamientos según el diagnóstico y sus relaciones clonales.

La mayoría de los aislamientos se encontraron relacionados genéticamente con el clon 3España ${ }^{9 v}$ y al comparar su distribución según el diagnóstico de neumonía o meningitis no presentaron diferencias significativas $(p=0,146)$. Contrario a lo observado en los aislamientos relacionados con el clon 2-España ${ }^{6 B}$, que se recuperaron principalmente de pacientes con neumonía $(p=0,052)$ y en los aislamientos relacionados con el clon 26 -Colombia ${ }^{23 F}$, los cuales en su mayoría se aislaron de pacientes con diagnóstico de meningitis $(p=0,001)$.

\section{Discusión}

En Colombia, la resistencia a la penicilina en aislamientos invasores de $S$. pneumoniae recuperados de niños menores de 5 años se ha incrementado a través del tiempo; es así como en el periodo de 1994 a 1996 fue del 12\% (6) y aumentó hasta el 47,4\% en el 2003 (12). En el mundo, el incremento en la prevalencia de aislamientos de $S$. pneumoniae resistentes a la penicilina se ha asociado con la naturaleza clonal de ciertas cepas resistentes, las cuales se han establecido como clones endémicos $(1,13)$, o con la evolución local de aislamientos resistentes que, por medio de transformación genética y recombinación homóloga, adquieren marcadores de resistencia y por el efecto de la presión selectiva del antibiótico pueden proliferar y establecerse como una población clonal $(11,13)$.

Desde 1998, Castañeda et al. establecieron la circulación de los clones internacionales 1 y 3 en la población pediátrica colombiana, con alta resistencia a la penicilina, y un grupo clonal de tipo capsular $23 \mathrm{~F}$ con resistencia intermedia a este antibiótico (6), reconocido actualmente como el clon $26-C o l o m b i a^{23 F}$ por la red de epidemiología molecular del neumococo (The Pneumococcal Molecular Epidemiology Network, PMEN)(14); posteriormente, Vela et al. demostraron la circulación en Colombia del clon multirresistente 2-España ${ }^{6 B}$ (7). En este estudio, estos 4 clones representaron el $76 \%$ de los aislamientos resistentes a la penicilina, lo cual demuestra su permanencia en el tiempo y su asociación directa con la prevalencia de la resistencia a la penicilina en nuestro medio (figura 1), de forma similar a lo observado en otras regiones del mundo $(2,15)$.

Al comparar la distribución de los aislamientos de $S$. pneumoniae relacionados con clones internacionales con los dos estudios previos de vigilancia molecular realizados en Colombia $(6,7)$, se observa un aumento de los aislamientos

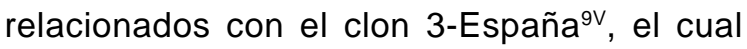
agrupaba el $12 \%$ de los aislamientos estudiados en el periodo de 1994-1996 (6) y, en la actualidad, comprende el $56 \%$ de los aislamientos resistentes a la penicilina (figura 1). Además, dentro de este grupo clonal sobresalen algunas subpoblaciones con tendencia a predominar, como el subtipo B3 que pasó de agrupar el 7\% de los aislamientos recuperados entre 1994 a 1996 (6) al 28\% de los aislamientos del presente estudio, posiblemente, como resultado de eventos genéticos que modificaron su contenido genético y, al mismo tiempo, facilitaron su proliferación y dispersión (16).

Previamente se había informado el serotipo 34 como variante capsular del clon 26 -Colombia ${ }^{23 F}$ $(6,7)$; no obstante, ninguno de los aislamientos estudiados con este serotipo se encontraron

Cuadro 2. Distribución de los aislamientos relacionados con clones internacionales según el diagnóstico.

\begin{tabular}{|c|c|c|c|c|c|c|c|c|c|}
\hline \multirow[t]{2}{*}{ Diagnóstico } & \multicolumn{2}{|c|}{ 1-España } & \multicolumn{2}{|c|}{ 2-España ${ }^{6 B}$} & \multicolumn{2}{|c|}{ 3-España ${ }^{9 v}$} & \multicolumn{2}{|c|}{ 26-Colombia ${ }^{23 F}$} & \multirow[t]{2}{*}{ Total } \\
\hline & $\mathbf{n}$ & $\%$ & $\mathbf{n}$ & $\%$ & $\mathbf{n}$ & $\%$ & $\mathbf{n}$ & $\%$ & \\
\hline Neumonía & 4 & 5,8 & 10 & 14,4 & 51 & 74 & 4 & 5,8 & 69 \\
\hline Meninigitis & 4 & 7,7 & 2 & 3,8 & 32 & 61,5 & 14 & 27 & 52 \\
\hline Otros & 1 & 3,8 & 2 & 7,7 & 23 & 88,5 & 0 & 0 & 26 \\
\hline
\end{tabular}

Otros: sepsis, peritonitis 


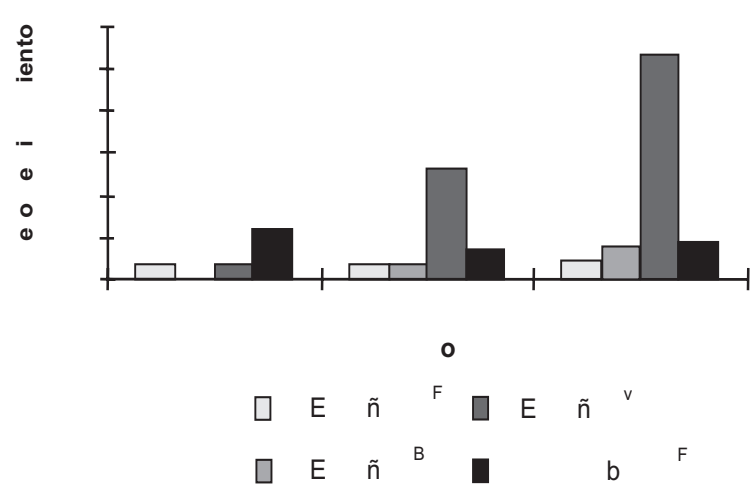

Figura 1. Distribución en el tiempo de los clones internacionales de Streptococcus pneumoniae en la población infantil colombiana.

relacionados genéticamente con este clon. Sin embargo, un aislamiento con serotipo $19 \mathrm{~F}$ se relacionó genotípicamente con el clon 26, posiblemente debido al intercambio de genes capsulares por recombinación homóloga que reemplaza parte del locus capsular $c p s$ del serotipo 23F por una región cps del tipo capsular 19F, de forma similar a lo observado con las variantes de serotipo $19 \mathrm{~F}$ del clon 1 -España ${ }^{23 \mathrm{~F}}(4,17)$.

Los aislamientos relacionados con los clones 1España $^{23 \mathrm{~F}}$ y 3 -España ${ }^{9 \mathrm{~V}}$ se recuperaron en similar proporción tanto de pacientes con meningitis como con neumonía, a diferencia de los aislamientos del clon 2-España ${ }^{6 B}$ que se recuperaron con mayor frecuencia de pacientes con neumonía y del clon 26-Colombia ${ }^{23 F}$ que en su mayoría procedían de pacientes con meningitis, pero no es posible establecer una relación entre un clon y una determinada entidad clínica debido al bajo número de aislamientos estudiados.

Los estudios de vigilancia molecular de los aislamientos de $S$. pneumoniae indican que la dispersión clonal es una de las principales causas de la prevalencia de resistencia a la penicilina en nuestro país, como resultado de la circulación de los clones internacionales 1-España ${ }^{23 F}$, 2España $^{6 \mathrm{~B}}$, 3-España ${ }^{9 v}$ y 26-Colombia ${ }^{23 F}$, en particular del clon 3 que es resistente a penicilina, ceftriaxona y trimetroprim-sulfametoxazol, y del clon 26 que se asocia con la dispersión de la resistencia intermedia a la penicilina. No obstante, no son la única causa debido a que el $23 \%$ de los aislamientos no se encontraron relacionados con clones internacionales; esto indica la posible transferencia horizontal de genes pbpque codifican para proteínas de unión a la penicilina con afinidad disminuida por el antibiótico $(12,13)$ o el surgimiento de nuevas líneas clonales resistentes que evolucionaron a partir de aislamientos locales en respuesta a la presión selectiva ejercida por la penicilina en nuestro país $(14,18)$.

Los estudios de vigilancia molecular han contribuido al mejor conocimiento de la epidemiología de las enfermedades infecciosas y son importantes para el control de la dispersión de la resistencia a los antibióticos que, además, de establecer la situación actual, también permiten evaluar el impacto de cualquier medida de intervención dirigida a disminuir la infección. Una de ellas es la inmunización de la población infantil con la vacuna heptavalente, la cual ha comprobado ser efectiva en la reducción de las infecciones invasoras en niños causadas por aislamientos resistentes a la penicilina y disminuir en los portadores nasofaríngeos los serotipos contenidos en la vacuna (19). Por consiguiente, la vacunación puede tener consecuencias en la distribución y prevalencia de grupos clonales, una consideración adicional que enfatiza la necesidad de mantener activa la vigilancia molecular de los aislamientos invasores de $S$. pneumoniae.

\section{Referencias}

1. Doern GV. Antimicrobial use and the emergence of antimicrobial resistance with Streptococcus pneumoniae in the United States. Clin Infect Dis 2001;33(Suppl.3):S187-92.

2. Ritcher SS, Heilmann KP, Coffman SL, Huynh HK, Brueggemann AB, Pfaller MA et al. The molecular epidemiology of penicillin-resistant Streptococcus pneumoniae in the United States, 1994-2000. Clin Infect Dis 2002;34:330-9.

3. Pfaller MA, Acar J, Jones RN, Verhoef J, Turnidge J, Sader. Integration of molecular characterization of microorganisms in a global antimicrobial resistance surveillance program. Clin Infect Diss 2001;32(Suppl.2): S156-67.

4. McGee L, McDougal L, Zhou J, Spratt G, Tenover FC, George R et al. Nomenclature of major antimicrobialresistant clones of Streptococcus pneumoniae defined by the Pneumococcal Molecular Epidemiology Network. J Clin Microb 2001;39:2565-71. 
5. Di Fabio JL, Castañeda E, Agudelo $\mathrm{Cl}$, De la $\mathrm{Hoz}$ F, Hortal M, Camou T, et al. Evolution of Streptococcus pneumoniae serotypes and penicillin susceptibility in Latin America, Sireva-Vigia Group, 1993-1999. Pediatr Infect Dis J 2001;20:959-67.

6. Castañeda E, Peñuela I, Vela C, Tomasz A. Penicillinresistant Streptococuus pneumoniae in Colombia: presence of international epidemic clones. Microb Drug Resist 1998;4:233-9.

7. Vela MC, Fonseca N, Di Fabio JL, Castañeda E. Presence of international multiresistant clones of Streptococcus pneumoniae in Colombia. Microb Drug Resist 2001;7:153-64.

8. National Committee for Clinical Laboratory Standards (NCCLS). Performance standards for antimicrobial susceptibility testing: twelfth information supplement. NCCLS document. Vol 22, no.1. Wayne, PA: National Committee for Clinical Laboratory Standards; 2002.

9. Soares S, Kristionsson KG, Musser JM, Tomasz A. Evidence for introduction of a multiresistant clone of serotype 6B Streptococcus pneumoniae from Spain to Iceland in late 1980s. J Infect Dis 1993;168:158-63.

10. Tenover FC, Arbeit RD, Gaering RV, Mickelsen PA, Murray BE, Persing DH et al. Interpreting chromosomal DNA restriction patterns produced by pulsed-field gel electrophoresis: criteria for bacterial isolate typing. J Clin Microb 1995;33:2233-9.

11. Coffey TJ, Dowson C, Daniels M, Zhou J, Martin C, Spratt $\mathbf{G}$ et al. Horizontal transfer of multiple penicillinbinding protein genes, and capsular biosynthetic genes, in natural populations of Streptococcus pneumoniae. Mol Microb 1991;5:2255-60.
12. Instituto Nacional de Salud. Datos de vigilancia epidemiológica (4 pantallas), consultado julio 06 de 2004. Disponible en http://www.ins.gov.co/pdf-investiga/mbitabla-1 pdf.

13. Tomasz A. New faces of an old pathogen: emergence and spread of multidrug-resistant Streptococcus pneumoniae. Am J Med 1999;107:55S-62S.

14. Pneumococcal Molecular Epidemiology Network (PMEN). Database. Accessed July 15, 2004. (4 pages). Available in: http://www.sph.emory.edu/PMEN/index.html.

15. Enright MC, Fenoll A, Griffiths D, Spratt BG. The three major Spanish clones of penicillin-resistant Streptococcus pneumoniae are the most common clones recovered in recent cases of meningitis in Spain. J Clin Microb 1999;37:3210-16.

16. Tenover F. Development and spread of bacterial resistance to antimicrobial agents: an overview. Clin Infect Dis 2001;33(Suppl.3):S108-15.

17. Coffey TJ, Enright MC, Daniels M, Morona JK, Morona R, Hryniewicz W et al. Recombinational exchanges at the capsular polysaccharide biosynthetic locus lead to frequent serotype changes among natural isolates of Streptococcus pneumoniae. Mol Microb 1998; 27:73-83.

18. Doit C, Denamur E, Picard B, Gelsin P, Elion J, Bingen E. Mechanisms of the spread of penicillin resistance in Streptococcus pneumoniae strains causing meningitis in children in France. J Infect Dis 1996;174:520-8.

19. Whitney CG, Farley MM, Hadler J, Harrison LH, Bennett NM, Lynfield R, et al. Decline in invasive pneumococcal disease after the introduction of proteinpolysaccharide conjugate vaccine. N Engl J Med 2003; 348:1737-46. 